\title{
Confidencialidad de datos en un hospital- escuela dedicado a la investigación
}

\author{
Elisa Hernández Rivera ${ }^{\text {- Patricia Raquel Mancilla Dávilab }}$
}

\begin{abstract}
Resumen: la información clínica que deriva de la atención médica es almacenada en los expedientes clínicos que se encuentran en archivos físicos o electrónicos en las distintas instituciones hospitalarias que se dedican a la enseñanza. Esta valiosa información constituye una herramienta fundamental en la investigación clínica, por lo tanto, debe de ser considerada confidencial desde que el paciente la remite hasta después de finalizar su tratamiento. Cuando los miembros de un hospital-escuela realizan actividades de investigación, utilizan la información contenida en los expedientes clínicos. La confidencialidad de estos registros es protegida por la ley, por esta razón es necesario fundamentar y desarrollar un método que ayude a los investigadores a conocer estos datos clínicos sin violar su privacidad y confidencialidad. Es necesario cuestionarnos si un aviso de privacidad que indique a los pacientes que la información derivada de sus padecimientos puede utilizarse posteriormente para fines de investigación será suficiente para resolver los conflictos relacionados con la obtención de datos. Un aviso de privacidad es un documento legal que se utiliza para la protección de datos de las personas, aplicarlo en las instituciones hospitalarias dedicadas a la enseñanza y a la investigación facilitará el uso de la información derivada de las consultas y mantendrá la confianza de que los datos utilizados en la investigación respetarán, la confidencialidad y la privacidad, a través del anonimato.
\end{abstract}

Palabras clave: aviso de privacidad; confidencialidad; datos personales; hospital; investigación

Recibido: 10/08/2020

Aceptado: 16/06/2021.

Disponible en línea: 31/12/2021.

a Magistra en Ciencia y Teconlogía de Alimentos, magistra en Bioética, licenciada de Químico en Alimentos. Instituto Mexicano de Oftalmología iap, Querétaro, México.

Correo electrónico: elisa.hernandez@imoiap.com.mx Código oRCiD: https://orcid.org/0000-0001-8565-581

b Magistra en Bioética, licenciada de Cirujana Dentista, Centro de Investigación Social Avanzada (CISAv), Querétaro, México.

Correo electrónico: patricia.mancilla@cisav.org Código oRCiD: https://orcid.org/0000-0003-4899-1926 
Cómo citar: Hernández Rivera E, Mancilla Dávila PR. Confidencialidad de datos en un hospital-escuela dedicado a la investigación. Rev. latinoam. bioet [Internet]. 31 de diciembre de 2021 [citado 31 de diciembre de 2021];21(2):41-55.

Disponible en: https://revistas.unimilitar.edu.co/index.php/rlbi/article/view/5111

\section{Data Confidentiality in a Hospital-School Dedicated to Research}

Summary: Clinical information derived from medical care is registered in clinical records in physical or electronic files in different hospital institutions dedicated to teaching. This valuable information is a fundamental tool for clinical research; therefore, it should be considered confidential from the moment the patient transfers it, until after the end of their treatment. When members of a hospital-school carry out research activities, they use information contained in clinical records. Confidentiality of these records is protected by law, for this reason, it is necessary to underpin and develop a method to help researchers to learn this clinical data without violating privacy and confidentiality. It is necessary to question ourselves whether a privacy notice indicating patients that the information derived from their conditions may subsequently be used for research purposes will be enough to resolve disputes related to data collection; in this regard, a privacy notice is a legal document used for the protection of people's data; using it in teaching and research hospital institutions will facilitate the use of information derived from consultations and maintain confidence that the data used in research will respect, first and foremost, confidentiality and privacy, through anonymity.

Keywords: privacy notice; confidentiality; personal data; hospital; research

\section{Confidencialidade de dados em um hospital-escola dedicado à pesquisa}

Resumo: As informações clínicas derivadas dos cuidados médicos são armazenadas nos prontuários médicos encontrados em arquivos físicos ou eletrônicos de diversos hospitais que se dedicam ao ensino. Essas informações valiosas são uma ferramenta fundamental na pesquisa clínica, portanto, devem ser consideradas confidenciais desde o momento em que o paciente as envia até o final do tratamento. Quando os membros de um hospital-escola realizam atividades de pesquisa, eles usam as informações contidas nos registros médicos. A confidencialidade desses registros é protegida por lei, por isso é necessário fundamentar e desenvolver um método que ajude os pesquisadores a conhecer esses dados clínicos sem violar sua privacidade e confidencialidade. É necessário questionar se um aviso de privacidade que informe aos pacientes que as informações derivadas de suas condições podem ser usadas posteriormente para fins de pesquisa será suficiente para resolver disputas relacionadas à coleta de dados. Um aviso de privacidade é um documento legal usado para a proteção de dados de indivíduos. Aplicá-lo em hospitais envolvidos em ensino e pesquisa facilitará o uso de informações derivadas das consultas e manterá a confiança de que os dados usados na pesquisa respeitarão, em primeiro lugar, a confidencialidade e a privacidade, por meio do anonimato.

Palavras-chave: aviso de privacidade; confidencialidade; dados pessoais; hospital; pesquisa 


\section{Introducción}

La investigación es un proceso sistemático que tiene por objetivo generar conocimiento actualizado de la realidad social, histórica, de mercado, de salud, etc. Las áreas de investigación son amplias y el avance tecnológico-científico ha permitido mayor acceso a la información de manera ágil y de fácil acceso.

La ciencia y la tecnología permiten el mejoramiento continuo y nutrido de un país, sociedad o institución. Aunado a la experiencia y aprendizaje diarios, la investigación se vuelve parte indispensable para el desarrollo profesional, social y educativo de toda institución dedicada a la enseñanza y formación de personal competitivo en el campo de la investigación. En el área de la salud, la promoción y fortalecimiento de la investigación se vuelve mayormente importante e indispensable debido a los avances acelerados en el área médica.

El desarrollo de investigaciones implica consultar grandes bases de datos, como expedientes clínicos digitales, fotografías, videos u otros archivos en los que se encuentran datos sensibles del sujeto de estudio. Sin embargo, el sujeto puede ser incluido dentro de un protocolo de investigación sin tener conocimiento del mismo.

Los hospitales-escuela tienen en su posesión datos sensibles de muchas personas que acuden día a día a consulta como pacientes, por eso es importante que las instituciones realicen estrategias eficientes para el tratamiento de datos personales con fines de investigación,

debido a que, sin que los individuos se enteren, ni mucho menos otorguen su consentimiento, terceros - ya sean entes públicos o privados- recaban y transmiten información sobre sus datos personales a través de todo tipo de procedimientos que echan mano de tecnologías de punta. [...]

No es posible concebir la vida de los seres humanos ni su interacción sin el uso de tecnologías informáticas urbi et orbi $[3,4]$. Dicha expansión conlleva el intercambio de flujos de información de todo tipo, incluida la relativa a las personas. $(1, \mathrm{p} 1)$
Los datos personales se definen como

toda información que atañe o es relativa a una persona física identificada o identificable a través de su nombre, edad, domicilio, sexo...; datos patrimoniales como cuentas bancarias, saldos, propiedades...; estados de salud físicos y mentales...; biométricos: huellas dactilares, iris, voz, firma autógrafa... Otros: ideología, afiliación política, religión, origen étnico, preferencia sexual... (2, p2)

El respeto a la privacidad de la persona, el resguardo de la confidencialidad y la protección de la información son cuestiones éticas distintas, pero relacionadas entre sí; vale la pena diferenciarlas, porque al violarlas las consecuencias y sanciones son distintas para los participantes (5). El aviso de privacidad es un instrumento que sustenta la vigencia del derecho fundamental de la protección de datos personales, que en esa calidad requiere ser elaborado y exhibido en todos los espacios en que se impliquen transacciones informativas y que debe ser conocido y comprendido por todas las personas al momento de decidir la entrega de algo tan valioso como son los datos que se relacionan con su individualidad $(6,7)$.

Para un hospital-escuela, tener, actualizar o generar un aviso de privacidad de protección de datos personales que hagan referencia a que los datos recolectados del paciente podrán utilizarse para fines de investigación manteniendo la confidencialidad, privacidad y el anonimato, puede ayudar a fortalecer procesos con fines educativos y científicos.

Por ejemplo, en México, la Ley General de Protección de Datos Personales en Posesión de los Particulares señala:

Art. 8 Todo tratamiento de datos personales estará sujeto al consentimiento de su titular, salvo las excepciones previstas por la presente Ley. El consentimiento será expreso cuando la voluntad se manifieste verbalmente, por escrito, por medios electrónicos, ópticos o por cualquier otra tecnología, o por signos inequívocos. Se entenderá que el titular consiente tácitamente el tratamiento de sus datos, cuando habiéndose puesto a su disposición el aviso de privacidad, no manifieste su oposición. Los datos financieros o patrimoniales requerirán el consentimiento expreso de su titular, salvo 
las excepciones a que se refieren los artículos 10 y 37 de

la presente Ley. (3)

Es obligación de toda institución mexicana tener a disposición del público en general el aviso de privacidad que especifique que se cuenta con bases de datos y que la información contenida en estas se encuentra blindada y protegida para la seguridad de los usuarios de los servicios de salud. En consecuencia, los datos que se recopilan y que se relacionan con la salud de cada paciente pueden utilizarse de forma anónima en la investigación científica con finalidad epidemiológica, estadística, cuantitativa, deductiva, no experimental, académica o de mercado, entre muchas otras. Esa valiosa información que se obtiene de primera mano a través del expediente clínico facilita el avance de la investigación médica, pues al interpretar los procesos de salud y enfermedad y aportar referencias directas sobre los tratamientos otorgados por cada institución, pueden mejorar los servicios de salud que otorga el establecimiento.

Este tipo de investigación que se realiza directamente sobre los datos en resguardo y que, por lo general, no requiere de una intervención directa con el paciente, puede dar la percepción errónea de tener la autoridad para omitir el consentimiento de los pacientes para que se revisen sus datos, por lo que se corre el riesgo de considerar inofensivo el acceder al expediente para obtener la información. Es necesario recordar que es deber del médico manejar responsablemente la información que el paciente le comunica (8).

Por otra parte, exponer el aviso de privacidad, conforme a la legislación correspondiente, fortalece la confianza de algunos usuarios para otorgar su información a la institución. Esto implica que suponer que no todos los usuarios de los servicios de salud leen el aviso de privacidad, no exime a la institución de publicarlo.

\section{Investigación educativa}

Actualmente, los modelos educativos que incluyen a los profesionales del área de salud tienen su fundamento en el aprendizaje basado en problemas y competencias como vías para mejorar la calidad del aprendizaje, es decir, en un programa educativo interdisciplinario.

La Medicina Basada en Evidencia (MBE) es una manera de integrar la competencia clínica individual como la evidencia disponible a partir de la investigación sistemática y con los valores y situaciones particulares del paciente. Su objetivo es ayudar al personal de salud a mejorar la toma de decisiones en la práctica clínica (9). La MBE juega un papel importante en la educación del profesional de salud en tres aspectos: la identificación del problema, la búsqueda de información sobre el mismo y la resolución del problema con relación a la información recogida (9).

Otro tipo de investigación es la relacionada con la obtención de información del expediente clínico. Estos expedientes son de gran utilidad para obtener hallazgos, especialmente de tipo epidemiológico, y obtener resultados con vías a mejorar la calidad en los servicios de salud. Sin embargo, aunque este tipo de investigación parezca inofensiva por no realizar una intervención física directa, sí se debe custodiar y manejar responsablemente toda información que el paciente revele al médico (10).

El profesional de salud se enfrenta entonces a nuevos retos, tanto en la práctica como en la enseñanza médica y ética. Actualmente, educar al estudiante del sector de salud para brindar atención integral centrada en la persona se añade al tema de la investigación como parte de la formación médica básica. Generar e implementar nuevos modelos educativos de calidad que fomenten el desarrollo científico-tecnológico, sin dejar de lado las responsabilidades éticas, requiere de un gran esfuerzo por parte de los formadores, comités de ética y directivos de instituciones privadas y públicas.

La investigación educativa es importante ya que aporta ventajas como: a) la continuidad con la que los pacientes asisten a la consulta, porque permite el seguimiento de sus estudios a largo plazo, b) la posibilidad de realizar estudios comunitarios, c) observar y dar seguimiento al amplio abanico de patologías que se atienden día a día, lo que brinda a los investigadores la oportunidad de abarcar numerosos ámbitos de especialización, d) la atención multidisciplinar, e) la accesibilidad integral a la 
información clínica de los pacientes y f) la posibilidad de realizar un trabajo colaborativo con otros centros de atención (10-11).

Respetar la privacidad y la confidencialidad se vuelve un imperativo ético, una obligación para todo el personal que desee realizar una investigación utilizando las bases de datos físicas o electrónicas existentes. Esto incluye las muestras obtenidas y almacenadas con otro fin, fotografías que puedan identificar a los pacientes o cualquier otra imagen o información digital almacenada en la que el consentimiento se haya obtenido para realizar cualquier otra investigación.

En casos especiales en los que no se pueda obtener la autorización y en casos que impliquen un riesgo, se tendrá que recurrir a un Comité de Ética en Investigación (CEI) para que determine si los intereses de los pacientes se verán comprometidos con el uso de la información, si existe o no un conflicto de interés o presión de alguna índole para obtener datos que favorezcan al investigador. El CEI determinará los elementos que componen la privacidad y confidencialidad de datos para evitar el conflicto de intereses y la violación a la privacidad y confidencialidad, además de ponderar el riesgo de un proyecto de investigación de tipo cualitativo-retrospectivo (12).

Es recomendable que, ante la incertidumbre, la falta de consenso o de normas al respecto, se dictamine que los protocolos de investigación sean evaluados por un CEI, pues en su metodología incluyen únicamente la revisión de expedientes.

\section{La ética aplicada a los nuevos modelos educativos en un hospital}

Fue durante la década de 1960 cuando unos maestros, médicos de origen canadiense, proponen modificar el tipo de enseñanza tradicional positivista de docencia-asistencia, por uno de tipo constructivista que propone integrar conocimiento, habilidades y actitudes a la enseñanza de la medicina (13).

Actualmente, la enseñanza y la educación médica han tomado un ritmo acelerado por los avances tecnológicos, el desarrollo del internet, la facilidad del acceso a la información al alcance de los dedos, la creación de nuevas máquinas que facilitan los diagnósticos y tratamientos médicos, que han permitido actualizar los métodos de enseñanza-aprendizaje en medicina. Sin embargo, estas nuevas competencias no deben desviar a los estudiantes ni a los prestadores de servicios de salud de su profesionalismo ético. Más bien, están llamados a rescatar los valores y principios originales de la medicina, como los plasmados en el juramento hipocrático, y los códigos deontológicos que se han acuñado a lo largo de la historia y que han sido renovados por la Bioética para salvaguardar la dignidad del paciente en la práctica médica. Los cambios en los modelos educativos han permitido dejar atrás el paternalismo médico y han abierto el paso a la autonomía $(14,15)$.

Haciendo hincapié en la investigación clínica como parte del modelo educativo, se observa que la información utilizada con fines de investigación se obtiene de diversas fuentes, en especial, de los expedientes clínicos (16) que incluyen el consentimiento informado. La investigación impulsada por datos ha abierto nuevas posibilidades para el descubrimiento científico, pero también conlleva riesgos de violar los principios bioéticos, entre ellos, el de autonomía. La mayor expresión de autonomía es tangible con la aplicación del consentimiento válidamente informado. Este consentimiento tiene origen a partir de los juicios de Núremberg $(17,18)$, cuando se descubrieron los experimentos médicos que llevaban a cabo los nazis en contra de la voluntad de las personas. Desde entonces, se ha normado para que el consentimiento informado sea un requisito indispensable en la práctica médica clínica y en la investigación biomédica.

La ética, unida a los nuevos modelos educativos, toma especial relevancia por el uso de las nuevas tecnologías y la práctica de la telemedicina, lo que presenta nuevos retos, especialmente, en lo relacionado a la obtención del consentimiento informado, la confidencialidad de datos, el aviso de privacidad, la protección de los datos personales, el almacenamiento de información personal en grandes bases de datos, el resguardo, la seguridad y la garantía de la confidencialidad de estos y el derecho a la intimidad (19). 
Los dilemas éticos en torno al derecho a la intimidad, definida como la esfera personal que está exenta del conocimiento generalizado de terceros (20), y el derecho de libertad informática médica o derecho a la autoderminación informativa constituye un aspecto debatible entre la inquietud del empleo de herramientas big data y los intereses científicos, políticos y financieros que se derivan de un uso malintencionado, manipulación o invasión a la privacidad $(20,21)$.

La Asamblea General de la Organización de las Naciones Unidas (ONU) explica "los principios rectores para la reglamentación de los ficheros computarizados de datos personales" (22, p200), entre los que se encuentran el principio de licitud y lealtad, el principio de exactitud, el principio de finalidad, el principio de acceso a la persona interesada, el principio de no discriminación, la facultad de establecer excepciones, el principio de seguridad, así como de control y sanciones, de flujo de datos a través de las fronteras y del campo de aplicación.

Estos principios son de especial interés al momento de consultar expedientes electrónicos y físicos, pues el acceso y uso de información debe preservarse para no poner en riesgo la identidad, la seguridad, la salud y la vida de cualquier persona $(23,24)$.

En algunos casos, el acceso a la información y publicación de datos en revistas científicas y académicas requieren que la persona otorgue voluntariamente su consentimiento. El dueño de estos sigue siendo la persona y tiene el derecho a solicitar el acceso cuando así lo desee. Un ejemplo de lo anterior es el caso de publicaciones denominadas como publicación de un caso o de casos clínicos en la que se exponen enfermedades poco comunes, nuevas enfermedades, complicaciones del tratamiento o resultados no esperados durante el tratamiento, con fines científicos y educativos. En estos casos se hace una descripción detallada del paciente, incluso se incluyen fotografías o imágenes, por lo que se debe especificar que se solicitó el consentimiento informado para la exposición de estas. Es importante señalar que la persona tiene derecho a no querer compartir los datos y esto no debe ser una razón para sufrir ningún tipo de discriminación ni falta de acceso a los servicios de salud (25).
El quehacer de la ética con respecto a proteger la privacidad y la confidencialidad es primordial en toda práctica dirigida a la investigación de cualquier persona. Es de considerar que los cei tienen una especial tarea para resguardar los derechos humanos relacionados a la intimidad y la protección de datos personales. Muchas veces las leyes de los Estados, que norman asuntos de privacidad y confidencialidad de datos en investigación, son inexistentes, insuficientes, poco claras o con lagunas.

En México, la Ley que regula estos temas es la Ley Federal de Protección de Datos Personales en Posesión de los Particulares (LFPDPPP). Entre otras cosas, indica que el Aviso de Privacidad es un mecanismo para recabar de manera responsable y efectiva el uso de datos personales (3):

Aviso de privacidad: toda persona física o jurídica (moral) que tenga acceso a datos personales de un particular, ya sea para su uso o para su explotación, con motivo de su actividad profesional, empresarial o comercial, se encuentra obligado a solicitar si quiere cualquier información personal, hacerle saber al particular titular de dicha información, qué información ha de pedirle, para qué necesita dicha información, así como qué fin o fines le ha de dar, es decir, su tratamiento, de igual manera, está obligado a proteger información personal que recabe o utilice, mediante protocolos y mecanismos de actuación. (26)

El acceso a la información para fines de investigación o de actividad profesional no siempre se encuentra bien definido o regulado. ¿En qué caso(s) es imperioso solicitar el consentimiento informado y en cuál(es) no? ¿Las publicaciones de tipo retrospectivo, epidemiológicas o sin intervención crítica deben ser exentas de ser revisadas por un CEI? Todo protocolo o trabajo de investigación, con fines científicos o educativos, por más inofensivo que pueda parecer, y que tenga de por medio la información de las personas, deberá someterse a un cei. A veces las normas no son suficientes para garantizar la revisión de metodologías con acceso a información electrónica o física y no existe la garantía para salvaguardar la información de todas las personas $(15,27)$. 


\section{La multidisciplinariedad para lograr objetivos}

En la actualidad, en México la investigación científica se ve muy limitada, tanto por los recursos económicos como por el personal profesional. Como lo señala Jimenez-Ponce: "[l]os hospitales públicos, en ocasiones, desarrollan algún proyecto para incrementar la formación de recursos humanos en ciencia, pero son escasos. (...) en los hospitales privados, la formación de investigadores es aún menos frecuente" (27, p173). En el mejor de los casos, los esfuerzos consistirán en cursos o diplomados temáticos que permitan una actualización del personal de salud.

En Latinoamérica, los estudiantes reconocen la importancia de la publicación científica, sin embargo, la estimulación y la enseñanza en el ámbito investigativo presenta dificultades, como la falta de interés y de constancia. Desafortunadamente, el poco interés del mismo docente es un factor influyente que pone en evidencia las deficiencias en programas educativos que promueven la realización de proyectos de investigación. Sánchez-Duque et al. señalan que la investigación científica durante el pregrado es un pilar fundamental durante la residencia del estudiante en una institución médica reconocida (28).

En las instituciones educativas de nivel superior y en las residencias médicas recae una mayor responsabilidad de educar con bases científicas y éticas para fomentar una práctica clínica y académica competitiva (29). Se resalta que la docencia y la investigación constituyen dos pilares necesarios para el desarrollo de las instituciones sanitarias (están relacionadas al sistema de salud) para garantizar el derecho del enfermo a la intimidad personal y familiar (30).

Generar la conciencia y la capacitación de cada uno de los investigadores, médicos adscritos, estudiantes, administrativos y, en general, de todo el personal relacionado con la obtención, recopilación y resguardo de los datos personales de pacientes en materia de seguridad y privacidad de la información es crucial para evitar errores que comprometan la difusión de los datos por cuestiones del sistema informático y escrito de la organización. Se deben "vigilar y actualizar los hábitos de seguridad del personal con acceso a los sistemas informáticos de la organización sanitaria" (31, p217).

La cooperación, la vinculación y la colaboración internacional, nacional, estatal e interinstitucional es necesaria para fortalecer la investigación, el desarrollo y el conocimiento científico. Sin embargo, países en vías de desarrollo presentan diferentes problemas de tipo geográfico, de logística y de infraestructura, para sostener o participar en investigaciones. Cuando la investigación se convierte en multicéntrica y la información compartida, si no se tiene un especial cuidado en ello, podría incurrirse en violaciones a la identidad y anonimidad del paciente, así como en conflictos de intereses por obtener más información de la estipulada. Nuevamente, la participación de los CEI es vital para asegurar la dignidad, los derechos, la seguridad y el bienestar de los participantes. Se reitera que la ética debe regir toda investigación como parte primordial y, de manera particular, en un hospital-escuela al que acuden muchas personas con la confianza de que sus datos personales no serán violados $(32,33)$.

Lo anterior no debería ser una limitante para el desarrollo profesional con base en el aprendizaje por competencias, sino que nos invita a una mayor reflexión, análisis y toma de conciencia por parte de todo el personal institucional involucrado en corporaciones que realizan cualquier tipo de investigación. Es mediante un equipo de trabajo con valores que se logra el cuidado del personal y de los pacientes, así como resguardar la información confidencial $(31,34,35)$.

Esta actitud de análisis y reflexión debería comenzar por el personal directivo, ya que, como cabeza de toda institución hospitalaria-educativa, propone los lineamientos y las estrategias para lograr que todos los departamentos trabajen en conjunto y se genere la conciencia del resguardo de datos personales que son sensibles para el paciente. Lo anterior se inicia desde la obtención de datos cuando se le pide al paciente el nombre para agendar la cita, hasta el llenado del historial clínico, la toma de fotografías diagnósticas, la petición de participar en un protocolo de investigación, etc. Además, los hospitales-escuelas que realizan 
investigación con bases de datos deberán implementar una logística que permita a los investigadores acceder a los datos que son de su interés, sin violentar la privacidad de los datos de los pacientes.

En muchas ocasiones, los datos de interés para un investigador no solo son la información con datos sensibles, sino también la información general contenida en las grandes bases de datos de las instituciones, como la prevalencia de enfermedades, el contexto social o demográfico, la epidemiología, la edad o el género. Por ello, la implementación de un flujo adecuado de acceso a las plataformas institucionales en las que se encuentran datos sensibles y generales requiere de una educación y conciencia ética de que el acceso no puede ser de uso indiscriminado y que, además, debe estar controlado, previa autorización de los responsables, para salvaguardar la información digital. De ahí que surja la necesidad de generar otra herramienta que complemente la seguridad de las grandes bases de datos: un aviso de privacidad. Por consiguiente, es importante que toda institución hospitalaria-educativa implemente o actualice, según sea el caso, su aviso de privacidad y, así, resguarde la información de los pacientes y, a la vez, sea de ayuda al investigador para que obtenga información, sin violar la intimidad de las personas y se apegue a las normativas vigentes de cada nación.

Por estas razones, el área jurídica debe participar activamente en elaborar o actualizar el aviso de privacidad conforme a las normas y reglamentos de cada país. Trabajando en equipo con la dirección médica, el departamento de calidad y el departamento de comunicación, se puede hacer que el aviso de privacidad sea posible, vigente, visible, legible y del conocimiento de toda persona que ingrese a las instalaciones hospitalarias. Por otro lado, el departamento de recursos humanos podría ser aquel que se encargue de la capacitación al personal sobre la importancia del aviso de privacidad de confidencialidad de datos, etc.

El departamento de enseñanza e investigación, como principal receptor y promotor de trabajos de investigación, será el primer beneficiario, pues será más efectivo el proceso para realizar la investigación, ya que no solo contarían con el respaldo de un comité de ética, sino también con un aviso de privacidad respaldado por la misma institución.

Contar con un aviso de privacidad actualizado, en el que se incluya un apartado que especifique que los datos recolectados en el expediente clínico, así como las fotografías, modelos de estudio, radiografías u otras imágenes almacenadas en el mismo y proporcionadas a la institución, pueden ser usados para fines científicos o educativos, resulta factible y seguro, si es reglamentado por la institución (36). También, es factible que el usuario de los servicios de salud, al estar enterado por medio del aviso de privacidad de confidencialidad de datos, niegue el derecho a usar estos datos. Si esto sucede, su expediente tendría que ser blindado o debe incluir una señal que especifique que el dueño de la información (es decir, el paciente) no ha autorizado el uso de sus datos ni con fines científicos ni educativos. Así no se podrá incluir su expediente en ninguna investigación de tipo retrospectivo o que incluya la revisión de los expedientes clínicos.

El aviso de privacidad con dicha especificación debería ser aplicable para todo hospital-escuela dedicado a la enseñanza y que, además, fomenta, realiza y es parte de los avances y conocimientos científicos. Para que la información contenida en el aviso de privacidad brinde los efectos deseados, debe ser útil, clara, transparente y entendible; además, debe mantenerse con el tiempo. También debe ser visible y del conocimiento de todo usuario y debe procurar abarcar el mayor número de medios posibles para que la información llegue al mayor número de personas usuarias del servicio hospitalario. Esto es de gran importancia, no solo porque se añade una garantía a la confidencialidad y privacidad de datos del paciente, sino que, además, es un medio para que el usuario mantenga la confianza en revelar información de carácter privado $(37,38)$.

Los retos que se presentan para lograr que el aviso de privacidad abarque la mayor población posible de pacientes y familiares que ingresan a un hospital-escuela son amplios. Reiteramos que la colaboración entre los diferentes departamentos o servicios dentro de cada institución es crucial. Se debe seguir un mismo objetivo: lograr que todo 
paciente que ingresa al instituto conozca el aviso de privacidad institucional. Esto puede lograrse mediante carteles visibles para los pacientes y familiares, incluir la leyenda en el carnet de citas y en el sitio web, también se puede colocar señalética que indique dónde puede localizarse el aviso de privacidad o pantallas que, mediante pautas informativas, mencionen de manera breve el uso de la recopilación y tratamiento de los datos personales. Poner en marcha este tipo de iniciativas requiere de, primero, un auténtico interés por salvaguardar la información de la persona y, segundo, un compromiso serio por parte de la institución, del personal sanitario y administrativo, con la seguridad y privacidad del paciente.

El beneficio, por lo tanto, es para ambas partes: educar e informa al paciente sobre el uso y tratamiento de sus datos podría ayudar a que la investigación institucional se vea favorecida cuando de investigaciones clínicas o experimentales se trate. De esa manera, el paciente sabrá que su participación, incluso indirectamente, es importante e impacta de manera positiva para realizar la investigación y contribuir al desarrollo científico. A este respecto, en un estudio realizado por Carbonell en el 2017 se analizó la percepción de los pacientes en la participación de ensayos clínicos (4, 39) y la mayoría de los pacientes están dispuestos a ser parte de una investigación, haciendo hincapié en que mantenerlos informados es una prioridad como participantes, tanto de los resultados que se obtuvieron hasta de un agradecimiento por su participación.

La percepción del paciente hacia la institución y el personal de salud, cuando es informado, es de confianza y es responsabilidad del personal y de la institución mantenerla. Una institución podría ser juzgada como buena cuando no solo responde a los intereses propios, sino también a los intereses y necesidades del paciente $(40,41)$.

Los retos a futuro giran en torno a concientizar a todo el personal médico, administrativo o que quiera hacer investigación con acceso a sistemas de base de datos particulares o públicos, para mantener, en todo momento, el resguardo y un uso respetuoso de los datos sensibles y generales de los pacientes y que, habiendo o no leído un aviso de privacidad, sigan sujetos a respetar toda clase de información.

\section{¿Un aviso de privacidad garantiza la seguridad del paciente?}

¿Es necesario realizar un nuevo consentimiento informado para usar los datos epidemiológicos o demográficos o para establecer la prevalencia de enfermedades?, ¿nos perderíamos de una investigación valiosa, tratando de recabar firmas?

Estas preguntas se podrían responder y justificar si en cada institución dedicada a la investigación con atención al paciente se trabajara con un aviso de privacidad de confidencialidad de datos. Así se crearía una base importante para mantener informados a los pacientes de aquellos datos que pueden usarse para beneficio de la sociedad y en pro de la investigación y del conocimiento científico, sin transgredir sus datos personales.

Tanto el término privacidad como confidencialidad están directamente relacionados y están explícitos bajos normas, sin embargo, son términos que implican valores no cuantitativos, como la virtud de la ética profesional, personal e institucional (42).

Lograr la infalibilidad y la efectividad de un aviso de privacidad y confidencialidad es algo muy subjetivo, pues se requiere de un esfuerzo de personas en el proceso. Implementar métodos que permitan de forma práctica y eficaz asegurar la protección de datos personales, pero que faciliten a los investigadores obtener la información necesaria y que aseguren el cuidado y la protección en el manejo de datos sensibles, son retos con los que un hospital-escuela se enfrenta de manera continua. Contar con comités de ética, aunado al aviso de privacidad, son algunas de las herramientas que ayudan a garantizar la privacidad y confidencialidad (42).

Es un deber mejorar la investigación en salud que favorezca al desarrollo científico, pero resguardando siempre la confidencialidad de datos del paciente. Por ejemplo, en México se tiene como fundamento la Norma Oficial Mexicana NoM-012SsA3-2012 (43), que establece los criterios para la 
ejecución de proyectos de investigación para la salud en seres humanos en los numerales 2 y 3; la Norma Oficial Mexicana NOM-004- SsA3-2012 (44), del Expediente Clínico; la Norma Oficial Mexicana NOM-024- SSA3-2012, Sistemas de información de registro electrónico para la salud, Intercambio de información en salud (45).

En otros países, como Costa Rica, la normatividad indica que el personal judicial debe mantener la confidencialidad de datos conforme a lo establecido en la Ley de Protección de la Persona frente al tratamiento de sus datos personales y el Reglamento de la Actuación de la Ley Frente al Tratamiento de sus Datos Personales en el Poder Judicial. Por otra parte, en Argentina, la Ley 25.326 describe ampliamente las disposiciones generales relativas a la protección, uso, responsabilidades, sanciones y acciones de protección de datos personales (46). Así, algunos países cuentan con políticas nacionales que protegen la integridad de toda persona en materia de ciencia, tecnología e innovación y en las cuales se involucra información contenida en grandes bases digitales nacionales e internacionales.

Los avances científicos nunca deben ser un pretexto para no salvaguardar la seguridad, los derechos y la intimidad de toda persona. Será de gran interés realizar o tener pautas internacionales respecto al uso de grandes bases de datos y que sirvan para realizar investigación de cualquier tipo. Hay que tener en cuenta que el principal riesgo de la investigación de tipo cualitativo, observacional y descriptivo es el daño a la información, la divulgación de la identidad de un grupo de personas, pueblos o etnias que pueden llevar a la discriminización y estigmatización social.

Una clasificación de la investigación conforme al riesgo que implica no es algo que se encuentre totalmente documentado. Generalmente se da por hecho que estudios de tipo sociológico, observacional o descriptivo no requieren de ser evaluados por instancias alternas, como un CEI, porque se piensa que no existen riesgos, daños al paciente o porque no se realiza ninguna intervención. En algunas ocasiones, el mismo investigador omite la revisión por un CEI justificando que se trata de un estudio de tipo observacional que no requiere intervención directa con el paciente y que, por lo tanto, no existen riesgos. La cuestión sería si realmente está libre de riesgos, cómo se asegurará y se comprometerá el investigador a mantener la confidencialidad de los expedientes clínicos que va a consultar, cuáles deben ser sometidos obligatoriamente a revisión por un comité de ética y cuáles no.

En México, aunque el Reglamento de la Ley General de Salud en Materia de Investigación para la Salud (47) en el Artículo 17 clasifica la investigación conforme al nivel de riesgo, no menciona la obligatoriedad de que sea revisada por un CEI. La clasificación conforme a la legislación mexicana es la siguiente: a) sin riesgo: cuestionarios, entrevistas, revisión de expedientes clínicos y otros, en los que no se identifiquen ni se traten aspectos sensitivos de la conducta del paciente, b) riesgo mínimo: procedimientos comunes en exámenes físicos o psicológicos de diagnósticos o tratamiento rutinarios o c) riesgo mayor que el mínimo, en que las probabilidades de afectar al sujeto son significativas, como ensayos con nuevos dispositivos, extracción del $2 \%$ de sangre en neonatos, entre otros (47). No debe eximir al investigador de presentar las consideraciones éticas pertinentes para todo tipo de trabajo de investigación, así sea desde revisar únicamente expedientes, hasta ensayos clínicos con un riesgo mayor.

Un aviso de privacidad, estipulado en leyes, es una herramienta adicional, pero no la única, que permite respetar la privacidad y la seguridad de los participantes. El aviso sí confiere protección al paciente, al investigador y a la institución donde se realiza la investigación, por ser de tipo normativo. Se debe continuar con el esfuerzo de que las leyes, reglamentos o normas de cada país ayuden a que los interesados en realizar investigación puedan tener un acceso responsable a las plataformas digitales con fines de divulgación, educación y avances científicos, pero teniendo la conciencia de que, de no respetar los acuerdos de confidencialidad o privacidad, las repercusiones pueden ser sancionadas por leyes que protegen la información de personas. 


\section{Reglamento de los bancos de datos médicos o personales para quienes investigan en un hospital-escuela}

Se propone un reglamento para regular las investigaciones que se realizan a partir de los expedientes clínicos, imágenes, radiografías, fotografías o bases de datos que se obtienen en los hospitales escuelas y que no han tenido una autorización o consentimiento previo e informado explícito para el uso y manejo de los datos personales sensibles por parte de los pacientes, quienes son dueños de la información. Este reglamento tiene el fin de ser implementado como parte de las políticas públicas.

1) Los datos que pueden ser utilizados en una investigación serán aquellos que fueron obtenidos con previo consentimiento del participante de la investigación. En caso de no contar con el consentimiento previo, el protocolo será sometido a un comité de ética que autorice la inclusión de los datos con fines de investigación.

2) Aquella institución que realiza investigación debe tener a la vista un aviso de privacidad y confidencialidad de datos con el fin de dar a conocer cómo se maneja la información.

3) Cuando se considere necesario, los participantes en la investigación firmarán un consentimiento informado amplio para el uso secundario de datos en investigación, que permitirá la reutilización de datos apegados a la recomendación ética del principio de respeto a la intimidad y confidencialidad. Se respeta la voluntad de aquellos pacientes que han negado su consentimiento para que sus datos participen en la investigación.

4) Dependiendo de los fines de la investigación, la información obtenida se clasificará en el siguiente rango: identificable, trazable o codificada, encriptada, anonimizada. Se trata del uso de un código seguro que proteja la confidencialidad de los participantes en la investigación.

5) La base de datos debe de tener códigos, contraseñas, claves y las medidas de seguridad que se requieren para garantizar la confidencialidad de las mismas y solo pueden ingresar a ellas los investigadores autorizados.

7) En ningún momento y bajo ninguna circunstancia los investigadores pueden contactar a las personas que han autorizado el uso de datos con el fin de recabar mayor información, sin que esta esté relacionada directamente con la investigación en curso.

8) El investigador no debe realizar un pago por los datos obtenidos, evitando así el conflicto de intereses.

9) El investigador o la institución a la que pertenece la investigación no puede comerciar con la información obtenida de los pacientes.

10) Los datos obtenidos de menores de edad no pueden ser utilizados en la investigación sin el consentimiento de los padres o tutores.

Esto es de especial interés, ya que en el instituto en que se realiza investigación de tipo retrospectivo-epidemiológico se ha llegado al dilema de si se puede o se debe obtener un nuevo consentimiento por el uso de los datos en resguardo.

Sin embargo, vemos que "la Comisión de Ética, por razones justificadas, podrá autorizar que el consentimiento informado se obtenga sin formularse por escrito y, tratándose de investigaciones sin riesgo, podrá dispensar al investigador la obtención del consentimiento informado" $(47, \mathrm{p} 7)$.

\section{Conclusión}

Todo Sistema Nacional de Salud (sNs) debería realizar investigación como una necesidad básica, pues renunciar a dicha actividad o dejarla en manos únicamente del sector industrial-farmacéutico es un sNs con una triste visión de futuro (29). Más que delimitar la práctica de la investigación, es ayudar a crear conciencia en el sector científico y de salud para que mantenga los principios éticos de privacidad y confidencialidad y, así, se eviten condicionamientos de crecimiento y desarrollo científico.

El riesgo que se corre por el acceso fácil y rápido a la información y al que se enfrentan los profesionales de la salud, los investigadores, las instituciones públicas o privadas es vigente y es un tema actual, asociado al mal uso de la información 
digital. El desarrollo tecnológico digital es importante, pero se puede caer en el error de no tomar en cuenta que todas las personas deben de contar con la garantía de que se respeten sus datos personales.

Perder de vista la privacidad y confidencialidad de los datos es una falta ética. Se debe tomar en cuenta que la ética, como disciplina, debe buscar el mayor bien moral y el mejor comportamiento del individuo en una adecuada conducta, con pilares y escalas de valores que, a pesar del paso de los siglos, se mantengan con la esencia del bien. Al respetar la información personal de nuestros semejantes, respetamos sus derechos y, por ende, sus garantías constitucionales y sus derechos humanos, reconocidos por los tratados internacionales, para evitar acciones discriminatorias o que menoscaben su integridad o dignidad, a causa de terceras personas.

Las instituciones de atención médica no están exentas de que los datos de los pacientes puedan ser violados, más cuando se dedican a promover y educar bajo un sistema que incluye la investigación. Implementar métodos de flujo y autorización de personas que ingresan al sistema institucional debe ser de especial interés como medio para garantizar la privacidad y confidencialidad de datos.

Garantizar la privacidad y confidencialidad, conforme a las normas de cada país, puede tener un impacto positivo en el área de la investigación para los hospitales-escuelas que, además, reciben residentes y estudiantes de medicina y de otras profesiones afines. Llegar a acuerdos nacionales e internacionales para clasificar investigaciones como sin riesgo, riesgo mínimo o riesgo mayor que el mínimo permitiría disminuir el riesgo de violar valores y principios éticos que afectan la integridad de los participantes.

La responsabilidad de mantener los principios bioéticos fundamentales en los hospitales-escuelas es amplia y exigente. Los modelos educativos exigen cada vez más el uso de tecnologías avanzadas, sofisticadas y de fácil acceso, sin embargo, esto no debe ser un pretexto para invadir y no respetar la privacidad y confidencialidad de cada dato o foto presente en los expedientes clínicos físicos y digitales. Lo anterior requiere de un trabajo en equipo con las diferentes áreas que integran la institución y que permita realizar investigación de calidad, profesional, ética y factible, sin perder de vista el valor moral que de ello se deriva. Destacamos la importancia de mantener actualizado a todo el personal colaborativo en temas de leyes y normas vigentes sobre la privacidad, la confidencialidad, los avances tecnológicos, etc., con fines de proteger la identidad, intimidad y seguridad de todo paciente-participante.

El desarrollo profesional en el sector salud, con base en los nuevos modelos educativos, es necesario, pero implica un gran desafío. Al tomar parte de la investigación en biomedicina, se enfrenta a esferas particulares, como el tratar con personas vulnerables por sufrir de alguna limitación o lesión física. El personal profesional de salud, sea docente, investigador, estudiante, etc., no debe perder de vista el respeto a la integridad del paciente. Por más novedosa o relevante que sea la investigación, la persona debe estar en primer plano. Complementar una educación basada en la ética y el respeto a los derechos y datos del paciente requiere de una capacitación constante $y$, así, mantener la integridad del paciente, sin renunciar o truncar la actividad de investigación.

Es importante destacar la intervención de la parte jurídica para realizar o actualizar un aviso de privacidad, pues esta parte es responsable de mantener actualizados los documentos legales, con base a leyes y normas que garanticen la privacidad y confidencialidad del paciente. Toda autoridad jurídica debe vigilar y garantizar la confidencialidad y protección de datos personales, así como cuidar que los intereses institucionales no se vean afectados por una mala praxis científico-ética.

Es necesario realizar una investigación más a fondo sobre cuántos hospitales-escuela tienen o incluyen un aviso de privacidad, normas o procedimientos sobre el manejo de datos para fines de investigación y evaluar la efectividad de los mismos: ¿el aviso es visible para el paciente?, ¿cuántos pacientes que acuden a las instituciones hospitalarias realmente saben que su datos pueden ser usados para fines de divulgación científica?, ¿cuántas personas conocen un aviso de privacidad de la institución? Estas y otras preguntas pueden surgir al momento de realizar una investigación exhaustiva sobre el uso, los procedimientos 
y el anonimato de la información contenida en las grandes bases de datos.

Un aviso de privacidad como único medio para responder a la seguridad personal del paciente no es garantía de que los datos del paciente o participante en un protocolo de investigación sean totalmente protegidos, por lo que toda institución dedicada a la investigación debe complementarlo con otras formas de resguardar y proteger información.

\section{Referencias}

1. Peschard J. La protección de datos personales en México: una propuesta para deliberar. IFAI [Internet]. 2008 Jul;1-11. Disponible en: http://iaipoaxaca.org.mx/biblioteca_virtual/datos_personales/5.pdf

2. Iaipchiapas.org. [Internet]. Chiapas (México): Instituto de Acceso a la Información Pública del Estado de Chiapas, Datos personales y redes sociales; s.f. [visitada en 2018 Jul]. Disponible en: https://www.iaipchiapas.org. $\mathrm{mx} / \mathrm{pdfs} /$ datos-personales/DP-Estudiantes.pdf

3. Ley Federal de Protección de Datos Personales en Posesión de los Particulares [Internet]; 2010 [visitada en 2018 Jul]. Disponible en: http://www.diputados.gob. $\mathrm{mx} /$ LeyesBiblio/pdf/LFPDPPP.pdf

4. Carbonell LA, García-Milian AJ, López-Puig P. La perspectiva del paciente del ensayo clínico. Rev Cub Sal Púb. [Internet]. 2017 Jun 27;43(3):373-95. Disponible en: http://www.revsaludpublica.sld.cu/index.php/ spu/article/view/792

5. Santi MF. Controversias éticas en torno a la privacidad, la confidencialidad y el anonimato en investigación social. Rev Bioét Der [Internet]. 2016;0(37):5-21. Disponible en: https://doi.org/10.1344/rbd2016.37.16147

6. Tenorio-Cueto GA, Rivero del Paso M. Los datos personales en posesión de los particulares. Análisis de su protección en México. Ars Iuris. [Internet]. 2011;(45):229-52. Disponible en: https://hdl.handle. net/20.500.12552/993

7. Cano-Guadiana A. Aviso de privacidad: un instrumento clave en la protección de los datos personales. El Finan [Internet]. 2017 Ag 1. Disponible en: https://www.elfinanciero.com.mx/opinion/areli-cano-guadiana/aviso-de-privacidad-un-instrumento-clave-en-la-proteccion-de-los-datos-personales/

8. Olaeta-Elizalde R. La importancia del expediente clínico. Rev Hosp Juá Méx [Internet]. 2001;68(2):95-103. Disponible en: https://www.medigraphic.com/pdfs/ juarez/ju-2001/ju012j.pdf
9. Junquera LM, Baladrón J, Albertos JM, Olay S. Medicina basada en la evidencia (MBE): Ventajas. Rev Esp Cirug Oral Maxilofac. [Internet]. 2003;25(5):26572. Disponible en: https://doi.org/10.4321/S113005582003000500003

10. Bayarre-Vea H. Estado actual y perspectivas de la investigación científica en la Atención Primaria de Salud. Rev Cubana Med Gen Integr. [Internet]. 2010;26(2). Disponible en: http://scielo.sld.cu/scielo.php?scrip$\mathrm{t}=\mathrm{sci}$ _arttext\&pid=S0864-21252010000200001\&ln$\mathrm{g}=\mathrm{es} \& \mathrm{nrm}=\mathrm{iso}$

11. García-Alcaraz F, Alfaro-Espín A, Moreno-Sotos JL. Evaluación de Resultados de Salud: Panorama sobre el uso de medidas de Resultados de Salud basadas en el paciente en la práctica clínica. Rev Clin Med Fam [Internet]. 2009;2(6):286-93. Disponible en: https://doi.org/10.4321/S1699-695X2009000100007

12. Welie JVM. Agenciamiento del paciente, autonomía y consentimiento. Perspectivas católicas. Rev Med Ética. [Internet]. 2020 Oct 1;31(4):803-42. Disponible en: https://doi.org/10.36105/mye.2020v31n4.03

13. Cash R, Wikler D, Saxena A, Capron A., editores. Estudios de casos sobre ética de la investigación internacional en salud. Washington D. C.: Organización Mundial de la Salud; 2014.

14. Pinilla AE. Modelos pedagógicos y formación de profesionales en el área de la salud. Acta Méd Colomb [Internet]. 2011 Ag 4;36(4):204-18. Disponible en: https://doi.org/10.36104/amc.2011.1451

15. García-Ortega C, Cózar-Murillo V, Almenara-Barrios J. La autonomía del paciente y los derechos en materia de información y documentación clínica en el contexto de la Ley 41/2002. Rev Esp Sal Púb [Internet]. 2004;78(4):469-79. Disponible en: https://doi.org/10.1590/S1135-57272004000400005

16. Farmacopea.org.mx [Internet]. Secretaría de Salud; s.f. [visitada en 2018 Jul]. Disponible en: https://www.farmacopea.org.mx/Repositorio/LegislacionFiles/Nom-004-SsA3-2012_15oct12.pdf

17. Ordovás-Bainesa JP, López-Brizb E, Urbieta-Sanzc E, Torregrosa-Sánchez R, Jiménez-Torres V. Análisis de las hojas de información al paciente para la obtención de su consentimiento informado en ensayos clínicos. Med Clin (Barc) [Internet]. 1999;112(3):904. Disponible en: http://esteve.org/wp-content/ uploads/2018/01/136883.pdf

18. Hernández-Mier C, Torre-Delgadillo V. Regulación del acceso al expediente clínico con fines de investigación en México. Rev conamed [Internet]. 2017;22(1):27-31. Disponible en: https:/www.medigraphic.com/pdfs/ conamed/con-2017/con171f.pdf 
19. López-Fernández W, Fernández-García A. Conocimientos de estudiantes de medicina sobre Bioética y medicamentos. 16 de Abr. [Internet]. 2020 Mzo 27;59(275):96-97. Disponible en: https://www.medigraphic.com/pdfs/abril/abr-2020/abr20275f.pdf

20. Hall R. Ética de la investigación social. Ciudad de México: Universidad Autónoma de Querétaro, Comisión Nacional de Bioética; 2017.

21. Litewka S. Telemedicina: un desafío para América Latina. Acta Bioeth [Internet]. 2005;11(2):127-32. Disponible en: https://doi.org/10.4067/S1726569X2005000200003

22. Robledo del Castillo et al. Régimen general. Protección de datos personales. Industria y Comercio Superintendencia; 2012.

23. Saab-Carrillo MD, Vinces-Fortún DS. Análisis jurídico del derecho a la intimidad [trabajo de grado]. Guayaquil: Universidad Católica de Santiago de Guayaquil; 2020.

24. Ramírez-García HS, Pallares-Yabur PdJ. Prólogo de Ángela Aparisi Miralles. Oxford University Press, México, 2011, 406 pp. Rev Pers Der. [Internet]. 2012;66:229249. Disponible en: https://revistas.unav.edu/index. php/persona-y-derecho/article/view/3133/2916

25. González E. El derecho a la salud. En: Abramovich V, Añón MJ, Courtis C, compiladores. Derechos sociales instrucciones de uso. Distribuciones Fontamara; 2006.

26. Pineda-Leguízamo R, Miranda-Novales G, Villasis-Keever MÁ. La importancia de los reportes de casos clínicos en la investigación. Rev Aler Méx [Internet]. 2018;65(1):92-98. Disponible en: https://doi. org/10.29262/ram.v65i1.348

27. Jiménez-Ponce F. La investigación científica en los hospitales privados. Acta Méd Grupo Áng. [Internet]. 2017;15(3):173-4. Disponible en: https://doi. org/10.35366/74380

28. Sánchez-Duque JA, Gómez-González JF, Rodríguez-Morales AJ. Publicación desde el pregrado en Latinoamérica: dificultades y factores asociados en estudiantes de Medicina. Invest Educ Méd [Internet]. 2017;6(22):104-8. Disponible en: https://doi.org/10.1016/j.riem.2016.07.003

29. Vinyoles E, Morralla C. Gestión de la Investigación. En: Diogene-Fadini J editor. Guía de investigación clínica para atención primaria. Barcelona: Ediciones Mayo, s.A.; 2005. p. 115-126.

30. Igualada-Menor A. La Ley de Protección de Datos y la comunicación científica. Medifam [Internet]. 2003;13(3):7-10. Disponible en: https://doi.org/10.4321/ S1131-57682003000300001
31. Sánchez-Henarejos A, Fernández-Alemán JL, Toval A, Hernández-Hernández I, Sánchez-García AB, Carrillo de Gea JM. Guía de buenas prácticas de seguridad informática en el tratamiento de datos de salud para el personal sanitario en atención primaria. Aten Prim. [Internet]. 2014;46(4):214-22. Disponible en: https:// doi.org/10.1016/j.aprim.2013.10.008

32. Macías-Chapula CA, Mendoza-Guerrero J, Rodea-Castro I, Juárez-Sánchez E, Gutiérrez-Carrasco A. Actividades de investigación y desarrollo en hospitales de América Latina y el Caribe, identificadas a través de sus sitios web. Rev Esp Docum Cient [Internet]. 2007;30(4):503-522. Disponible en: https://doi. org/10.3989/redc.2007.v30.i4.402

33. Avendaño C, Casas A, Dal-Ré R, Gomis R, Gracia $\mathrm{D}$, Moreno $\mathrm{A}$, et al. Comités éticos de investigación clínica y "dictamen único" de los ensayos clínicos multicéntricos. Med Clín [Internet]. 2003;120(5):180188. Disponible en: https://doi.org/10.1016/S00257753(03)73643-4

34. Cano-González R. Tutoría universitaria y aprendizaje por competencias, ¿cómo lograrlo? Rev Elect Interuniv Form Profes [Internet]. 2009;12(1). Disponible en: https://dialnet.unirioja.es/servlet/articulo?codigo $=2956810$

35. Fernández-March A. La evaluación orientada al aprendizaje en un modelo de formación por competencias en la educación universitaria. REDU Rev Docenc Univ [Internet]. 2011;8(1):11-34. Disponible en: https://doi. org/10.4995/redu.2010.6216

36. Imoiap.com.mx. Querétaro: Oftalmología IMD; 2019. [visitada en 2019 Sept] Disponible en: https://imoiap. com.mx

37. Barbero J. El derecho del paciente a la información: el arte de comunicar. Anales Sis San Nav [Internet]. 2006;29(S3). Disponible en: https://doi.org/10.4321/ S1137-66272006000600003

38. García-Millán Á. La información al paciente como pieza clave de la calidad asistencial. Rev Clin Med Fam [Internet]. 2009;2(6):275-279. Disponible en: https:// doi.org/10.4321/S1699-695X2009000100005

39. Romero-Ameri LC. Percepción del paciente acerca de la calidad de atención que brinda la enfermera en el servicio de medicina en el Hospital Nacional Daniel Alcides Carrión [Trabajo de grado]. Lima: Universidad Nacional Mayor de San Marcos; 2008.

40. Delgado-Marroquín MT. Título de Experto en Ética Médica. 06 Confidencialidad y secreto profesional. Organización Médica Colegial de España, Fundación para la Formación, Fundación Ortega-Marañón; 2012. 
41. Miret RC, Espeleta LB. El secreto médico. En: González MC, coordinadora. Bioética y derecho y sociedad. Madrid: Editorial Trotta; 1998. p. 151-182.

42. Ríos-Ortega J, Ramírez-Velázquez CA, coordinadores. Uso ético de la información: implicaciones y desafíos. 1.a ed. Ciudad de México: Universidad Nacional Autónoma de México; 2017.

43. Norma Oficial Mexicana NOM-012-ssA3-2012 [Internet]; 2012 [visitada en 2018 Jul]. Disponible en: http:// dof.gob.mx/nota_detalle.php?codigo $=5284148 \&$ fecha $=04 / 01 / 2013$

44. Norma Oficial Mexicana Nom-004-ssA3-2012 [Internet]; 2012 [visitada en 2018 Jul]. Disponible en: https:// www.farmacopea.org.mx/Repositorio/LegislacionFiles/Nom-004-Ssa3-2012_15oct12.pdf

45. Norma Oficial Mexicana NOM-024-ssA3-2012 [Internet]; 2012 [visitada en 2018 Jul]. Disponible en: http:// www.dgis.salud.gob.mx/descargas/normatividad/ normas/DOF-30NOV12-NoM-024-SsA3-2012.pdf

46. Valesani ME, Mariño SI, La Red Martínez D. La Protección de los datos personales en los sistemas informáticos. La instrumentación en la Argentina. cisic [Internet]. 2003. Disponible en: https://www. researchgate.net/publication/228718681_La_Proteccion_de_los_Datos_Personales_en_los_Sistemas_Informaticos_La_Instrumentacion_en_la_Argentina

47. Reglamento de la ley general de salud en materia de investigación para la salud [Internet]; 2014 [visitada en 2020 Sept]. Disponible en: http://www.diputados.gob. $\mathrm{mx} /$ LeyesBiblio/regley/Reg_LGS_MIS.pdf 